\title{
Evaluation of Connexin 43 Redistribution and Endocytosis in Astrocytes Subjected to Ischemia/Reperfusion or Oxygen-Glucose Deprivation and Reoxygenation
}

\author{
Hongyan Xie, ${ }^{1}$ Yu Cui, ${ }^{2}$ Shuai Hou, ${ }^{3}$ Juan Wang, ${ }^{1}$ \\ Jing Miao, ${ }^{3}$ Fang Deng, ${ }^{3}$ and Jiachun Feng ${ }^{3}$ \\ ${ }^{1}$ Department of Neurology, Affiliated Hospital of Taishan Medical University, Taian 271000, China \\ ${ }^{2}$ Department of Neurosurgery, Affiliated Hospital of Taishan Medical University, Taian 271000, China \\ ${ }^{3}$ Department of Neurology, The First Hospital of Jilin University, Changchun 130021, China \\ Correspondence should be addressed to Fang Deng; 409841537@qq.com and Jiachun Feng; fengjcfrank@126.com
}

Received 9 December 2016; Revised 23 February 2017; Accepted 8 March 2017; Published 23 March 2017

Academic Editor: Esmaiel Jabbari

Copyright (c) 2017 Hongyan Xie et al. This is an open access article distributed under the Creative Commons Attribution License, which permits unrestricted use, distribution, and reproduction in any medium, provided the original work is properly cited.

Connexin $43(\mathrm{Cx} 43)$ is the major component protein in astrocytic gap junction communication. Recent studies have shown the cellular processes of gap junction internalization and degradation, but many details remain unknown. This study investigated the distribution of $\mathrm{Cx} 43$ and its mechanism after ischemic insult. Astrocyte culture system and a model of ischemia/reperfusion (IR) or oxygen-glucose deprivation and reoxygenation (OGDR) were established. Cx43 distribution was observed by laser scanning confocal microscopy under different cultivation conditions. Western blot and RT-PCR assays were applied to quantify Cx43 and MAPRE1 (microtubule-associated protein RP/EB family member 1) expression at different time points. The total number of Cx 43 was unchanged in the normal and IR/OGDR groups, but $\mathrm{Cx} 43$ particles in the cytoplasm of the IR/OGDR group were significantly greater than that of the normal group. Particles in the cytoplasm were significantly fewer after endocytosis was blocked by dynasore. There was no difference among the groups at each time point regarding protein or gene expression of MAPRE1. We concluded that internalization of $\mathrm{Cx} 43$ into the cytoplasm occurred during ischemia, which was partially mediated through endocytosis, not by the change of $\mathrm{Cx} 43$ quantity. Moreover, internalization was not related to microtubule transport.

\section{Introduction}

Stroke is a leading cause of death and long-term disability in humans. In central nervous system, astrocytes have diverse and important roles in many aspects of ischemic brain damage [1]. They form a glial network and communicate through gap junctions (GJs), allowing passage of ions and small metabolites among adjacent cells.

Vertebrate GJs consist of protein subunits termed connexins $(\mathrm{Cx})$ [2]. $\mathrm{Cx} 43$ is the only connexin expressed by cultured astrocytes that was shown firstly by Giaume and Dermietzel et al. in 1991 [3, 4]; it is endogenously expressed in at least 35 tissues and cell types and is a major component protein in astrocytic GJs [5-7]. Since astrocytes are deemed an important participant in ischemia, their quantity and alteration have recently been the focus of research [8]. Exploring such features and the underlying molecular mechanisms of Cx43 by using an in vitro ischemic model may help to determine therapeutic target(s) in ischemia.

The short half-life of Cx proteins (1-5 h) [9] indicates GJ transport should be a dynamic progress. Furthermore, the relatively rapid rate of turnover in astrocytes is essential for Cx43-mediated GJ intercellular communication [10]. Modulation of the synthesis and degradation rate of $\mathrm{Cx} 43$ may be an important way to control the level of GJs under various conditions [6,11]; regulation of GJs and Cx43 in astrocytes may answer to various stimuli like ischemia. Elevated levels of Cx43 immunoreactivity in astrocytic processes and cell bodies associated with brain ischemia have been demonstrated, and redistribution of $\mathrm{Cx} 43$ in myocardia answers to myocardial ischemia $[12,13]$. 
Shinotsuka et al. [14] reported that astrocytes provided protection against tissue damage by way of their GJ-mediated intercellular network after oxygen and glucose deficiency. It is beneficial to study whether protective effects of astrocytes are mediated via $\mathrm{Cx} 43$ redistribution, and we focused on $\mathrm{Cx} 43$ redistribution and used carbenoxolone (CBX) to block GJs in this study. CBX, a nonselective blocker of connexin and pannexin hemichannels [15], has been widely used to block GJs in several systems and inhibits cell death [16].

The balance between synthesis and degradation of a protein determines its steady-state level. Some evidences showed that $\mathrm{Cx}$ transport was mediated partially by microtubules that are intrinsically polar: the plus-end is the fastgrowing end and the minus-end is stable or serves as the site of disassembly [17]. Key to this process appears to be microtubule plus-end tracking proteins (+TIPs) like microtubule-associated protein $\mathrm{RP} / \mathrm{EB}$ family member 1 (MAPRE1) and its homologues [18]; changes in MAPRE1 may reflect the formation, transport, and decomposition process of the microtubule. Thus, we observed not only microtubule transport by detecting changes of MAPRE1 gene expression and protein quantity, under normal conditions, but also conditions of ischemia/reperfusion (IR) or oxygen-glucose deprivation and reoxygenation (OGDR).

GJs are retrieved from the cell membrane to the cytoplasm for degradation [9]. However, whether this progress involves endocytic machinery needs to be determined [9]. There are approximately 10 different endocytic pathways coexisting in mammalian cells, and the majority of them are regulated by dynamin $[19,20]$. Dynamin is a member of a superfamily of GTPases, which can pinch off invaginated membrane pits. Dynasore is a noncompetitive inhibitor of dynamin GTPase activity and blocks dynamin-dependent endocytosis in cells [21]. In the present study, Cx43 distribution was observed under IR/OGDR condition by using CBX or dynasore.

\section{Materials and Methods}

2.1. Astrocyte Cultures. The experiments were approved by the Experimental Animal Research Committee of Jilin University and were performed according to the Guidelines for Animal Experimentation of Jilin University. Primary astrocyte cultures were prepared from the cortex of 1-dayold Wistar rats. Rats were decapitated, and the cortices were dissected and transferred to cold D-Hanks solution. The tissues were incubated in phosphate-buffered saline (PBS) containing $0.125 \%$ trypsin for $10 \mathrm{~min}$ at $37^{\circ} \mathrm{C}$. The reaction was terminated by Dulbecco's modified Eagle's medium/nutrient mixture F-12 containing 20\% fetal bovine serum (FBS; GIBCO, Grand Island, USA). The tissues passed through a $40 \mu \mathrm{m}$ cell strainer. Cells were centrifuged at $1500 \mathrm{rpm}$ for $10 \mathrm{~min}$. The resulting cell pellets were resuspended and seeded into culture flasks at a concentration of $1 \times 10^{5}$ cells $/ \mathrm{cm}^{2}$ in culture dishes. The medium was changed $2 \mathrm{x} /$ week. When astrocytes in flasks reached confluence, they were separated into 2 dishes for further culture for 3-4 weeks. The final cultures containing $>95 \%$ astrocytes and $<5 \%$ microglia were verified for the following experiments.
2.2. In Vitro Model of Ischemia/Reperfusion (IR) or OxygenGlucose Deprivation and Reoxygenation (OGDR). An in vitro model of IR/OGDR was made by incubating astrocyte cells in glucose-free Earle's balanced salt solution and oxygen deprivation media (bubbled with 5\% $\mathrm{CO}_{2} / 94.9 \% \mathrm{~N}_{2} / 0.1 \% \mathrm{O}_{2}$ for $30 \mathrm{~min}$ ). Then reperfusion was initiated in normal media (DMEM/F12 containing $10 \% \mathrm{FBS}$ ) at $37^{\circ} \mathrm{C}$ in 5\% CO2/95\% air atmosphere at time points $0.5,1,3,6,12,24$, and $72 \mathrm{~h}$.

2.3. Cell Survival Assessment. Cell survival was assessed by MTT (3-[4,5-dimethylthiazol-2-yl]-2,5-diphenyltetrazolium bromide) assay. Cells were cultured in 96-well plates at a density of 10,000 cells/well. After a $36 \mathrm{~h}$ treatment with various concentrations of CBX $(5 \mathrm{mM}, 1 \mathrm{mM}, 500 \mu \mathrm{M}, 200 \mu \mathrm{M}$, $100 \mu \mathrm{M}, 50 \mu \mathrm{M}, 20 \mu \mathrm{M}$, and $10 \mu \mathrm{M}), 20 \mu \mathrm{L}$ of MTT solution $(5 \mathrm{mg} / \mathrm{mL})$ was added to each well and further incubated for $4 \mathrm{~h}$. The MTT solution was aspirated gently. DMSO $(150 \mu \mathrm{L} /$ well $)$ was added to the wells. The cells were incubated with shaking for $10 \mathrm{~min}$. The optical density of the supernatant was read at $570 \mathrm{~nm}$ (reference wavelength, $630 \mathrm{~nm}$ ) using a microplate reader (Bio-Rad, USA). Absorbance was normalized to the untreated control cultures, which represented $100 \%$ viability. The viability rate was calculated as viability: $\%=$ mean absorbance of sample/mean absorbance of control $\times 100 \%$. All data presented in this study were obtained from 5 independent experiments.

2.4. Immunofluorescence Staining. Cells were fixed in $-20^{\circ} \mathrm{C}$ cold methanol. After washing in PBS, cells were blocked with $10 \%$ nonimmune goat serum (Sigma, St. Louis, USA) for $30 \mathrm{~min}$ at room temperature and incubated with $0.1 \%$ (v/v) Triton X-100 (Sigma, St. Louis, USA) for $5 \mathrm{~min}$. The cells were serially incubated with primary antibodies at $4^{\circ} \mathrm{C}$ overnight. Control experiments were conducted under identical conditions, except that primary antibodies were replaced by PBS. Coverslips were incubated with the corresponding secondary antibody for $45 \mathrm{~min}$. The following primary antibodies were used: rabbit anti-Cx43 (1:1000, Abcam, Cambridge, UK), mouse anti-glial fibrillary acidic protein (GFAP, 1:500; Boster, Wuhan, China), and rabbit anti-MAPRE1 (1:500, Abcam, Cambridge, UK). Secondary antibodies consisted of a 1:500 dilution of donkey antirabbit IgG conjugated to fluorescein isothiocyanate (FITC) (1:250, ZSGB-BIO, Beijing, China) and goat anti-mouse IgG conjugated to FITC (1:250, ZSGB-BIO, Beijing, China). After incubation, Hoechst 33342 (Sigma, St. Louis, USA) was added; incubation time was $20 \mathrm{~min}$. All coverslips were observed under a laser-scanning confocal microscope (Olympus, Japan).

To evaluate changes in Cx43 distribution, we selected time points at which cell injury was observed to be the most severe, using MTT colorimetry. The cultures were divided into the following groups: normal culture, IR/OGDR saline, and IR/GDR with carbenoxolone (CBX) intervention. Observations were conducted using a confocal laserscanning microscope. After blocking endocytosis with dynasore, the change in redistribution of $\mathrm{Cx} 43$ was observed under the confocal laser-scanning microscope. The relative fluorescence intensity of the cell and nuclei in each group 
was measured by Image-Pro Plus 7.0 Software, to provide semiquantitative analysis.

2.5. Western Blot Analyses. The methods described by Bradford (1976) were employed to detect the total protein concentration of cells. Protein homogenates of astrocyte cultures were subjected to SDS-polyacrylamide gel electrophoresis and then transferred to a polyvinylidene fluoride (PVDF) membrane. After blocking, each membrane was incubated overnight at $4^{\circ} \mathrm{C}$ with rabbit anti-Cx43 (1:5000, Abcam, Cambridge, UK), anti-MAPRE1 antibody (1:500, Abcam, Cambridge, UK), or anti- $\beta$-actin antibody $(1: 10,000$, Sigma, St. Louis, USA). The membrane was then incubated with an IgG alkaline phosphatase-conjugated antibody $(1: 10,000$, Sigma, St. Louis, USA) for one hour at $37^{\circ} \mathrm{C}$. The membrane was washed, and MAPRE1 and $\beta$-actin were detected using a BCIP/NBT substrate system (Sigma, St. Louis, USA). The optical density of each protein band was measured with NIH Image $\mathrm{J}$ software and normalized as to the optical density of the corresponding $\beta$-actin. All samples were made in triplicate.

2.6. RNA Extraction and RT-PCR Assay. Cells were collected into a $1.5 \mathrm{~mL}$ Eppendorf tube by repeatedly pipetting with $1 \mathrm{~mL}$ Trizol. Added to this was $0.2 \mathrm{~mL}$ chloroform. These were vigorously oscillated and then placed at room temperature for $3 \mathrm{~min}$. The water phase was transferred to a new tube after centrifugation and kept at $-20^{\circ} \mathrm{C}$ for 20 min with the same volume of isopropyl alcohol. The RNA precipitate was collected after centrifugation. One $\mathrm{mL}$ of $75 \%$ ethanol was used to wash the RNA precipitate, which was placed at room temperature for about 5-10 min; RNase-free water was used to dissolve the RNA, and then it was preserved at $-70^{\circ} \mathrm{C}$. The amount of total RNA was measured using a NanoDrop 2000/2000c (Thermo Scientific, Dreieich, Germany). All samples were made in triplicate.

For each sample, total RNA was reversely transcribed into cDNA with a SuperScript II Reverse Transcriptase Kit (Invitrogen, USA). The reaction was allowed for $5 \mathrm{~min}$ at $30^{\circ} \mathrm{C}, 60 \mathrm{~min}$ at $42^{\circ} \mathrm{C}$, and $15 \mathrm{~min}$ at $72^{\circ} \mathrm{C}$. RT-PCR was performed using $20 \mu \mathrm{L}$ of a mixture composed of $10 \mu \mathrm{L}$ SYBR, $0.4 \mu \mathrm{L}$ S-Primer, $4 \mu \mathrm{L}$ AS-Primer, $0.4 \mu \mathrm{L}$ ROX, $1 \mu \mathrm{L}$

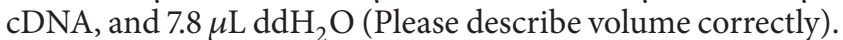
The reaction was performed in the IQ5 iCycler (Bio-Rad, Munchen, Germany) using the following 3-step amplification protocol: $30 \mathrm{~s}$ at $95^{\circ} \mathrm{C}$ (enzyme activation), 40 cycles of $5 \mathrm{~s}$ at $95^{\circ} \mathrm{C}$ (denaturation), $34 \mathrm{~s}$ at $61^{\circ} \mathrm{C}$ (annealing), and $15 \mathrm{~s}$ at $95^{\circ} \mathrm{C}$ and $60 \mathrm{~s}$ at $60^{\circ} \mathrm{C}$ (extension). RT-PCR products were resolved in $1.2 \%$ agarose by gel electrophoresis. Cycle threshold $(\mathrm{Ct})$ values were statistically analyzed using REST Software [22]. The sequences of the primers we used are as follows: rat MAPRE1 sense, $5^{\prime}$-AAG AGG ATG GGC GTT GAC A - $3^{\prime}$; and antisense, $5^{\prime}$-AAG GAG CCA CTG CCG TTT C- $3^{\prime}$; and rat $\beta$-actin sense, $5^{\prime}$-GAG GCC CCT CTG AAC CCT AA- $3^{\prime}$; and antisense, $5^{\prime}$-ACC AGA GGC ATA CAG GGA CAA- $3^{\prime}$.

2.7. Statistical Analysis. The data were analyzed using SPSS19.0 software. All data are shown as mean \pm standard deviation. Comparisons among 2 or more groups were performed by means of an independent-samples $t$-test and analysis of variance, followed by Fisher's partial least-squares difference. Differences with a $P$ value $\leq 0.05$ were considered statistically significant. The graphs were drawn by GraphPad Prism 6.0 software.

\section{Results}

3.1. Determination of CBX Concentration and Astrocytes in Response to IR/OGDR. The MTT colorimetric method was applied to analyze the cytotoxicity of CBX and to determine the optimal concentration. No obvious toxic effect on the survival of astrocytes was observed when CBX was at the concentration of $20 \mu \mathrm{mol} / \mathrm{L}$. Therefore, $20 \mu \mathrm{mol} / \mathrm{L}$ of CBX was used in this study. Cultured astrocytes were subjected to oxygen-glucose deprivation for $2 \mathrm{~h}$ and then to normal supplements of oxygen and glucose at $0.5,1,3,6,12,24$, or $72 \mathrm{~h}$. Astrocytes were most severely damaged, and viability was lowest $6 \mathrm{~h}$ after reperfusion and then gradually improved and returned to nearly normal level at $72 \mathrm{~h}$. Therefore, the time point $6 \mathrm{~h}$ after reperfusion was chosen for observation during the follow-up study.

3.2. Influence of IR/OGDR on Redistribution of Cx43. We made the quantitative analysis of $\mathrm{Cx} 43$ in the normal and at different time points of IR/OGDR groups. As shown in Figures 1(a) and 1(b), the total amount of $\mathrm{Cx} 43$ was unchanged under normal and IR/OGDR conditions $(P=$ 0.307). We analyzed the fluorescence intensity of $\mathrm{Cx} 43$ in astrocytes by Image-Pro Plus 7.0 Software and drew the graph by GraphPad Prism 6.0 software. As shown in Figure 1(c), the total level of $\mathrm{Cx} 43$ of astrocytes was unchanged among groups; only distribution occurred. This was consistent with the conclusion of Figures 1(a) and 1(b).

We examined the distribution of $\mathrm{Cx} 43$ with a laserscanning confocal microscope under normal and IR/OGDR conditions. As shown in Figures 2(a) and 2(b), it was found that there was no difference in the total amount of $\mathrm{Cx} 43$ $(P=0.761)$ between the normal and IR/OGDR groups; however, the cytoplasm particles of the IR/OGDR group were significantly greater in number than that of the normal group $(P=0.019)$. Furthermore, some vesicles of different sizes and shapes appeared in the cytoplasm of the IR/OGDR group, which were obviously distinct from the endoplasmic reticulum and Golgi apparatus transport vesicles in the normal group. Under IR/OGDR conditions with CBX intervention, which was shown in Figure 2(c), Cx43 redistribution was not affected $(P=0.217)$ compared with the saline control group.

3.3. Effect of Dynasore on Redistribution of Cx43. To block endocytosis, dynasore was used. In addition, DMSO, as a universal solvent to dissolve dynasore, was selected as the control group to rule out its influence. According to the literature, we knew that the final working concentration for in vivo experiments was $80 \mu \mathrm{mol} / \mathrm{L}(0.2 \% \mathrm{DMSO}$ final), which typically resulted in a greater than $90 \%$ blockage in endocytosis [21]. Therefore, we selected $80 \mu \mathrm{mol} / \mathrm{L}$ as the working concentration of dynasore. As shown in Figures 3(a) and 3(c), we found that Cx43 still moved from the cell 


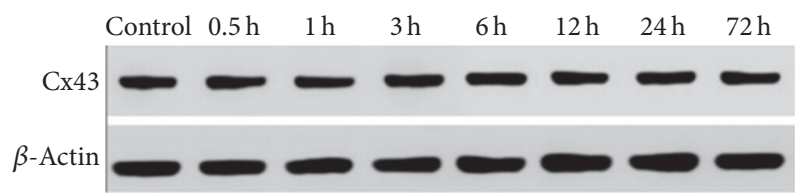

(a)

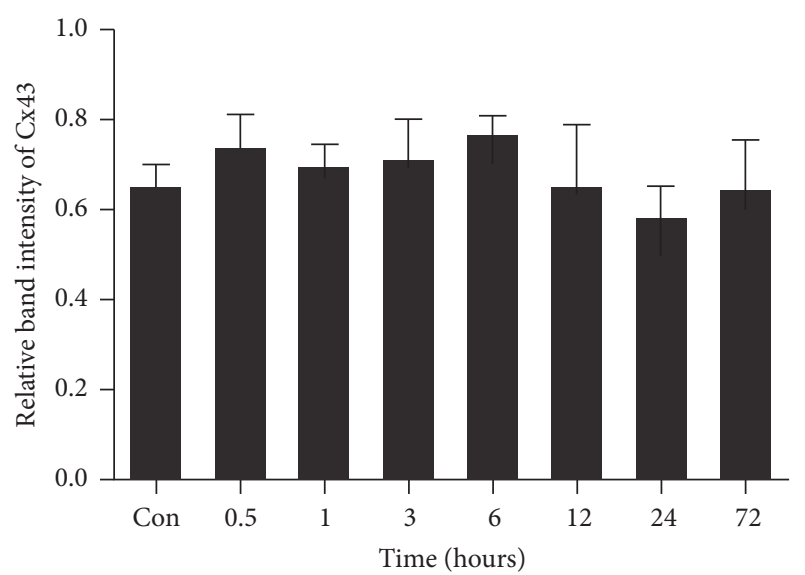

(b)

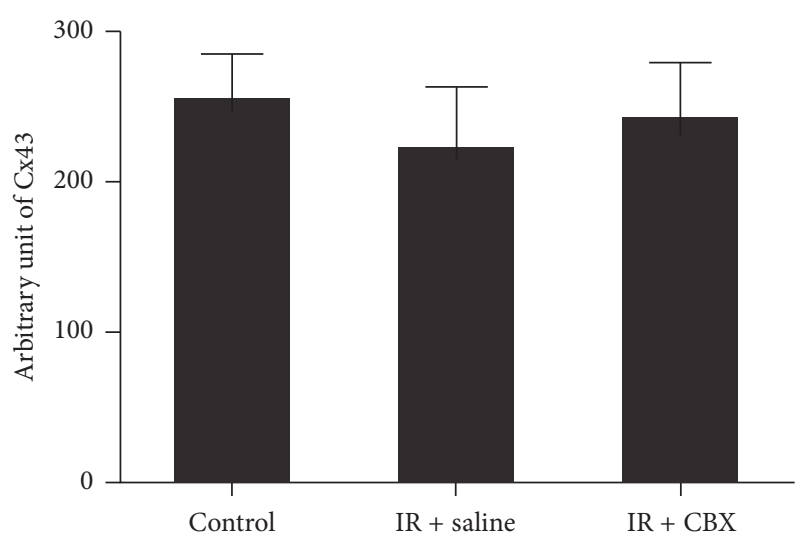

(c)

FIgURE 1: Representative western blot of $\mathrm{Cx} 43$ in the normal and at different time points of IR/OGDR groups. (a) The first stripe was $\mathrm{Cx} 43$ protein; the other was $\beta$-actin as the internal stripe. (b) $\mathrm{Cx} 43$ protein relative expression. There was no significant difference among the groups at each time point $(P=0.307)$. (c) Representative graph of fluorescence intensity value of $\mathrm{Cx} 43$ in normal and IR/OGDR $6 \mathrm{~h}$ (saline/CBX) groups. There was no significant difference among the three groups $(P=0.208)$.

membrane to the cytoplasm when endocytosis was blocked with dynasore. As depicted in Figures 3(b) and 3(c), the cytoplasm particles of the group under IR/OGDR conditions with dynasore were significantly fewer than those in the DMSO control group $(P=0.021)$, and the number of particles transported into the cytoplasm was reduced by $29.2 \%$.

3.4. No Significant Difference in MAPRE1 among All Groups under IR/OGDR Conditions. Western blot was applied for quantitative analysis of the MAPRE1 protein. There was no significant difference in the protein levels of MAPRE1 among the groups at different times $(P=0.69)$. Moreover, we analyzed the gene expression of MAPRE1 via RT-PCR, and no significant difference was observed among the groups at each time point $(P=0.634)$; however, its mRNA amount at $6 \mathrm{~h}$ was minimal (Figure 4). It is suggested that the transportability of microtubules was lowest at this time. These results showed that transport by microtubules may be reduced, indicating that Cx43 synthesized in the endoplasmic reticulum could not be transported efficiently to the cell membrane.

\section{Discussion}

In the present study, we showed that the total amount of $\mathrm{Cx} 43$ was unchanged under IR/OGDR conditions. However, some internalized vesicles that were different from endoplasmic reticulum or Golgi apparatus transport vesicles were observed in the cytoplasm of the IR/OGDR group. Moreover, CBX did not affect $\mathrm{Cx} 43$ redistribution. There was no statistical difference among the groups in protein levels or gene expression of MAPRE1. Particles in the cytoplasm in the dynasore intervention group were significantly fewer than in the control group under IR/OGDR conditions. We concluded that internalization of $\mathrm{Cx} 43$ into the cytoplasm occurred during ischemia, which was partially mediated through endocytosis, and not by a change in the number of $\mathrm{Cx} 43$ particles. Moreover, internalization was not related to microtubule transport.

Alterations in $\mathrm{Cx} 43$ expression and function are involved in the pathophysiology of some diseases, such as brain ischemia [23]. Nakase et al. [24] were the first to investigate the level of $\mathrm{Cx} 43$ expression in the human brain, finding that it increased under long-term ischemia. Downregulation of Cx43 significantly increased the survival of pyramidal neurons and improved cognitive impairment after middle cerebral artery occlusion (MCAO) [25]. However, Cx43 knockout mice showed a significantly increased brain stroke volume and enhanced apoptosis [26]. Orellana et al. showed that hypoxia-reoxygenation enhanced activity of $\mathrm{Cx} 43$ hemichannels and reduced gap junctional coupling of cortical astrocytes in culture, and these effects were potentiated by high as well as zero glucose during the hypoxic period [27]. Later, Orellana et al. also found that reoxygenation after $3 \mathrm{~h}$ hypoxia in high glucose induced transient astroglial permeabilization and reduction in intercellular communication via $\mathrm{Cx} 43$ based channels [28]. As the above conclusions were derived from different experimental systems, it is difficult to predict the roles of connexins in ischemia. Therefore, we used IR/OGDR model to mimic cerebral ischemia and observed the change of $\mathrm{Cx} 43$.

The present study showed that the total number of Cx43 was unchanged in the normal and IR/OGDR groups; however, some internalized vesicles were observed in the cytoplasm of the IR/OGDR group. Their size was smaller than that of organelles in the cytoplasm, which was observed roughly under a laser-scanning confocal microscope. The size of these vesicles could not be accurately measured due to the limitations of the experimental conditions, which required further experiments. Martinez and Sáez found that hypoxia/reoxygenation altered gap junctions and was 

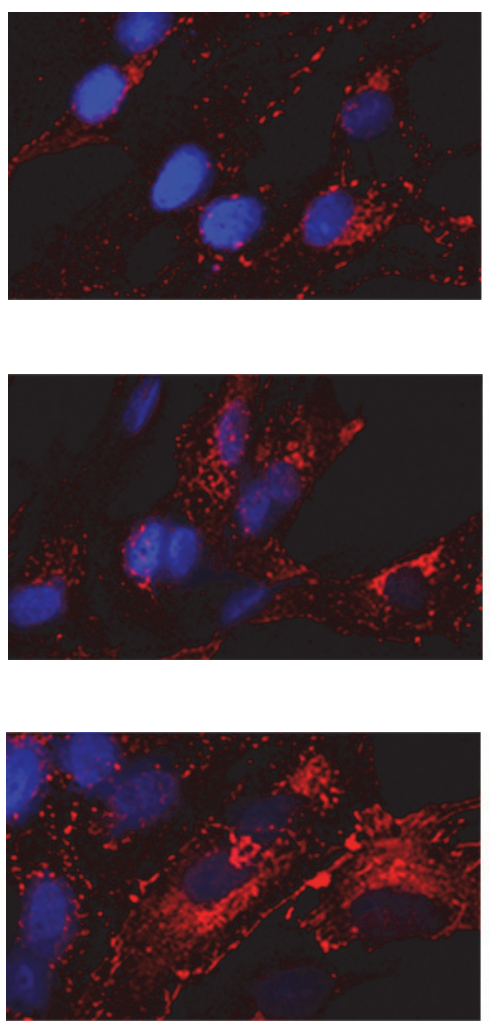

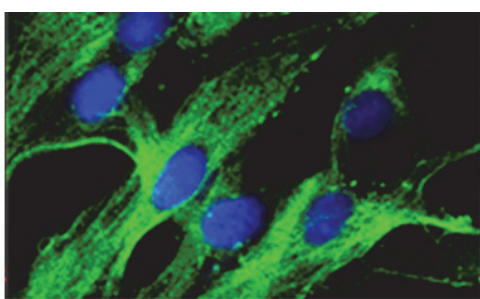

(a)

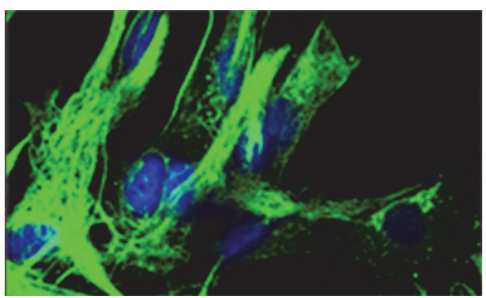

(b)
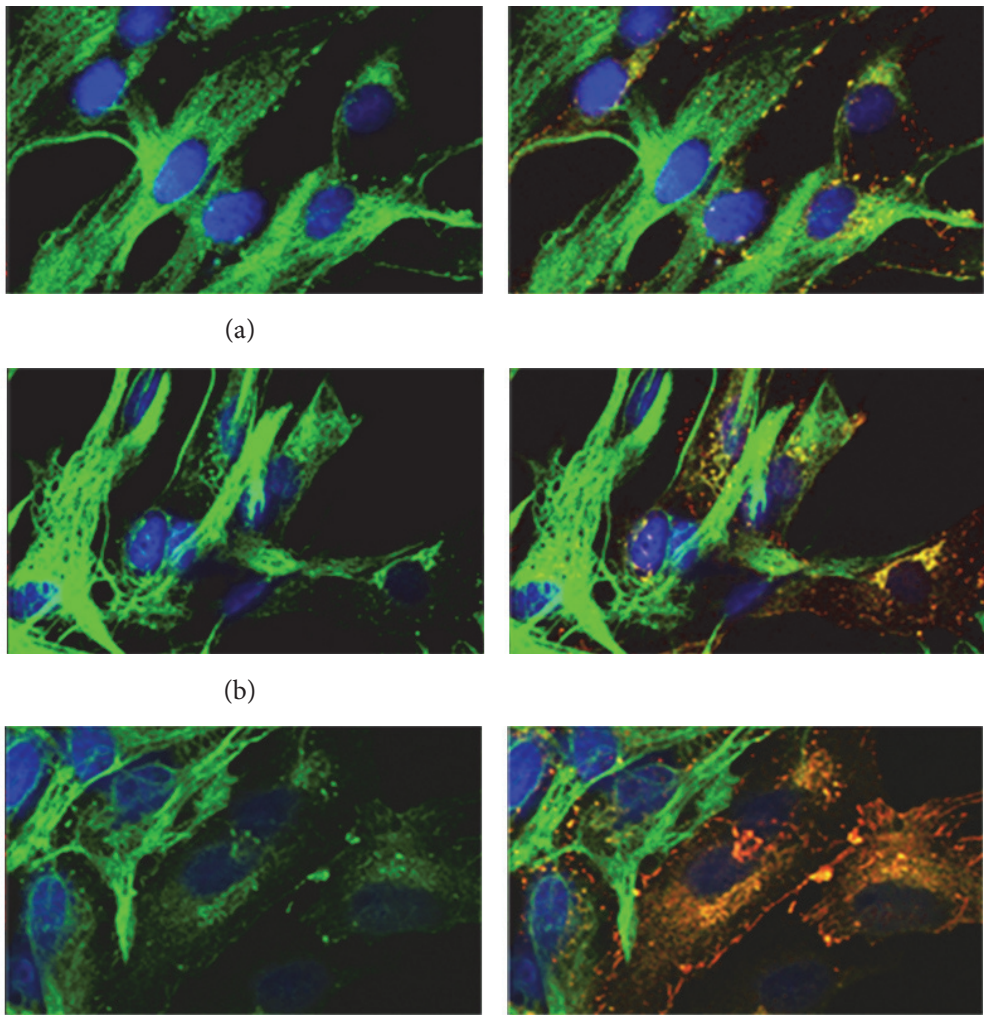

(c)

Figure 2: Representative confocal images of Cx43 distribution under different conditions at $6 \mathrm{~h}$ after reperfusion (red, Cx43; green, GFAP; blue, Hoechst 33342; magnification was 400x, resp.). (a) Under normal cultivation conditions, Cx43 was mainly distributed in the cytoplasmic membrane, and its density was higher in the adjacent cell membrane joint. A small amount of scattered Cx43 has been circulated in the cytoplasm with a few particles distributed in the nucleus. (b) Under IR/OGDR conditions with physiological saline, Cx43 was distributed in the membrane, cytoplasm, and nucleus, with the highest content in the cytoplasm. These vesicles of different sizes and shapes were observed in the cytoplasm. The total number of particles of this group was almost the same as that of the normal group $(P=0.761)$, but the cytoplasm particles of this group were significantly more than those of the normal group $(P=0.019)$. (c) Under IR/OGDR conditions with CBX intervention, $\mathrm{Cx} 43$ was distributed in the membrane, cytoplasm, and nucleus but mainly in the cytoplasm. There was no significant difference between the CBX intervention and saline control group $(P=0.217)$.

associated with cellular redistribution of proteins, which was believed to constitute a lysosomal pathway for $\mathrm{Cx} 43$ degradation [29]. Similar to this report, we also found that Cx43 was transported into the cytoplasm and nuclei during ischemia, which was consistent with the fact that transfected Cx43 was predominantly localized in the cytosol and nucleus [30]. Moreover, it has been reported that $\mathrm{Cx} 43$ could bind to DNA, suggesting that $\mathrm{Cx} 43$ has distinct functions from its well-known involvement in GJ intercellular communication [31]. Orellana et al. [32] showed that chlorpromazine did not induce obvious cell shape changes in astrocyte cultures but it clearly changed the distribution of $\mathrm{Cx} 43$, and this distribution was based on changes in the state of microtubules and microfilament. In agreement with this viewpoint, we analyzed the distribution of $\mathrm{Cx} 43$ and unchanged expression of MAPRE1 under normal and IR/OGDR conditions. Alternatively, it might be explained that the functions of gap junction channels were regulated by the state of $\mathrm{Cx} 43$ and MAPRE1 but not by their quantity.

As the licorice derivative, CBX can block almost all types of GJs regardless of Cx composition, and it is well tolerated at even high doses [33]. We used CBX at $20 \mu \mathrm{mol} / \mathrm{L}$, which was confirmed by an MTT assay, consistent with a previous report [34]. Frantseva et al. [35] found that CBX was neuroprotective but did not significantly alter intrinsic neuronal characteristics. Also, CBX does not penetrate the blood-brain barrier [36]. Therefore, for the treatment group in the present study, we applied CBX to block GJs, to examine whether its neuroprotective role involved $\mathrm{Cx} 43$. The results showed that CBX treatment did not change $\mathrm{Cx} 43$ redistribution. This suggested that the quantity was changed by $\mathrm{CBX}$, and it may invaginate into the cytoplasm to form utricle bubbles. Up to now, CBX is a well-known molecule that is able to inhibit some subtypes of gap junction proteins including $\mathrm{Cx} 43$ and gap-junctionrelated protein called pannexin-1 [37]. Moreover, CBX affects multiple targets, including voltage-gated calcium channels, intrinsic neuronal properties, and neurotransmitter release and acts as an anticonvulsant [38]. And thus it can be seen that CBX has a wide range of effects, which is also doomed to have many side effects such as cytotoxicity. Cytotoxicity of CBX on astrocytes was determined by performing the MTT assay. Relative to other concentrations of CBX, $20 \mu \mathrm{mol} / \mathrm{L}$ 

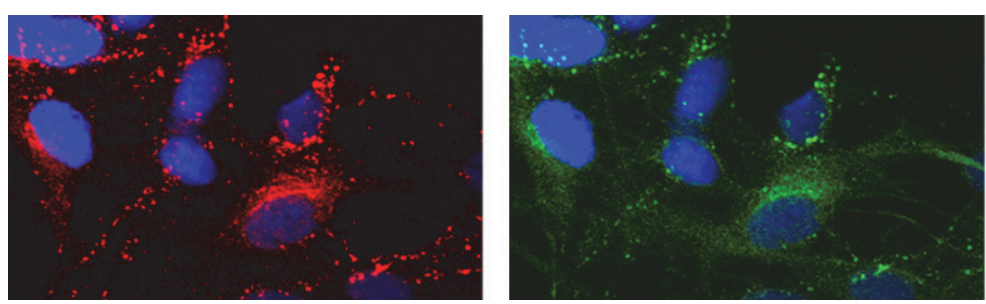

(a)
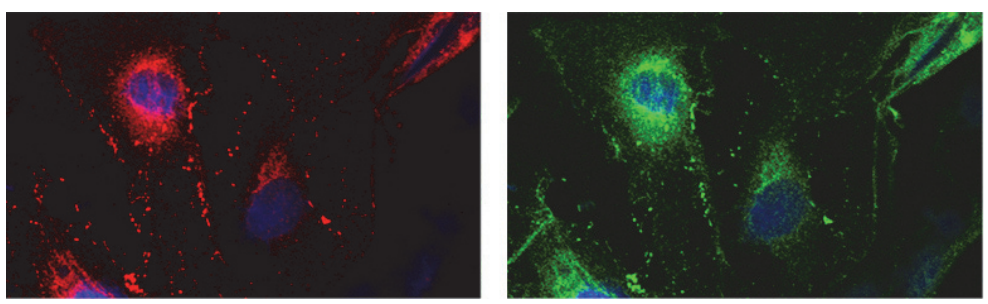

(b)
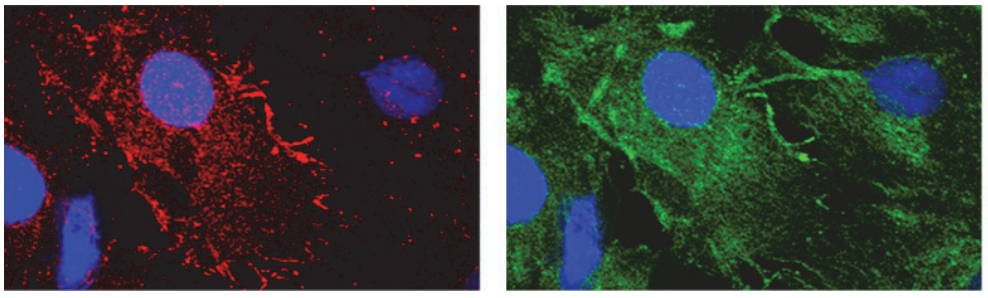

(c)
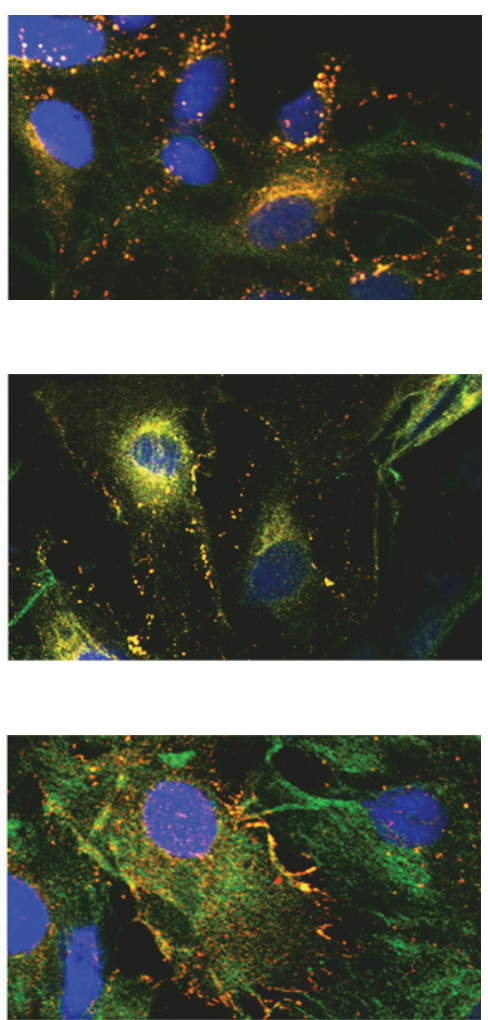

अ

)

FIGURE 3: Representative confocal images showed Cx43 distribution under IR/OGDR conditions at $6 \mathrm{~h}$ after reperfusion (red, Cx43; green, GFAP; blue, Hoechst 33342; magnification was 400x, resp.). (a) Under normal cultivation conditions, the distribution of Cx43 is similar to that in Figure 1(a). (b) Under IR/OGDR conditions with DMSO (as the medium to dissolve dynasore), the distribution of Cx43 was almost the same as that in Figure 2(b). (c) Under IR/OGDR conditions with dynasore, $\mathrm{Cx} 43$ was distributed in the membrane, cytoplasm, and nucleus, with the highest content in the cytoplasm. The particles in the cytoplasm of this group were significantly fewer than those of the DMSO control group $(P=0.021)$, and the number of particles transported into cytoplasm was reduced by $29.2 \%$.

did not cause cytotoxic effect on the survival of astrocytes. Meanwhile, this concentration of CBX could block gap junctional communication, $\mathrm{Cx} 43$ hemichannels, and pannexin 1 channels as well.

As the transport of $\mathrm{Cx} 43$ is mediated by microtubules, it is phosphorylated and oligomerized into hexameric structures termed connexon [39]. MAPRE1 functions in GJ formation, which promotes delivery of Cx to the cell-cell border [40]. Some studies have shown that microtubules were dispensable for the regulation of $\mathrm{Cx} 43$ [41]. Recent studies revealed that Cx43 modulated cytoskeletal proteins including MAPRE1, which are involved in process formation and migration of astrocytes. Besides, Stephan et al. found that MAPRE1 expression depended on the level of $\mathrm{Cx} 43$ expression by the method of immunocytochemistry and western blot [42]. In the present study, we showed that there was no difference in protein and gene expression of MAPRE1 after IR/OGDR at time points $0.5,1,3,6,12,24$, and $72 \mathrm{~h}$. In agreement with this viewpoint, we also found that the total number of $\mathrm{Cx} 43$ was unchanged in the normal and IR/OGDR groups. Although the levels of protein and gene expression of MAPRE1 were not different after IR/OGDR at every time point examined, it could be explained that the integrity or redistribution of microtubules and microfilament might be more relevant to the trafficking of $\mathrm{Cx} 43$ containing vesicles. Thereby, this report offered the chance to gain further insights into the mechanisms by which astrocytes could achieve distribution of Cx43 after ischemic insult.

Entire or fragments of GJs internalized as doublemembrane circular structures were traditionally called annular junctions [43]. A recent study revealed that annular junctions originated from preexisting gap junction plaques at cell-cell interfaces [44]. Gaietta et al. [45] showed that newly synthesized GJs are assembled from the outer edges of plaques, whereas aged channels were removed to the central core. These internalized GJs are large and structurally different from typical endocytic cargo [46] and are likely to be distinct from conventional endocytic processes, such as classical endosomes or phagosomes $[6,46]$. Recent studies found that some dynamin was recruited to the endocytic pathway to abstrict invagination of vesicles during evolution [47]. Gumpert et al. [48, 49] reported clathrin-mediated endocytosis, the most extensive route involving dynamin. Our results showed that internalization was reduced partially by dynamin. This supported the role of dynamin in GJ internalization. 


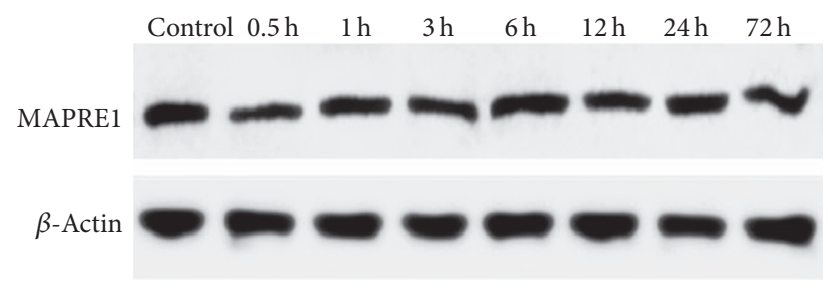

(a)

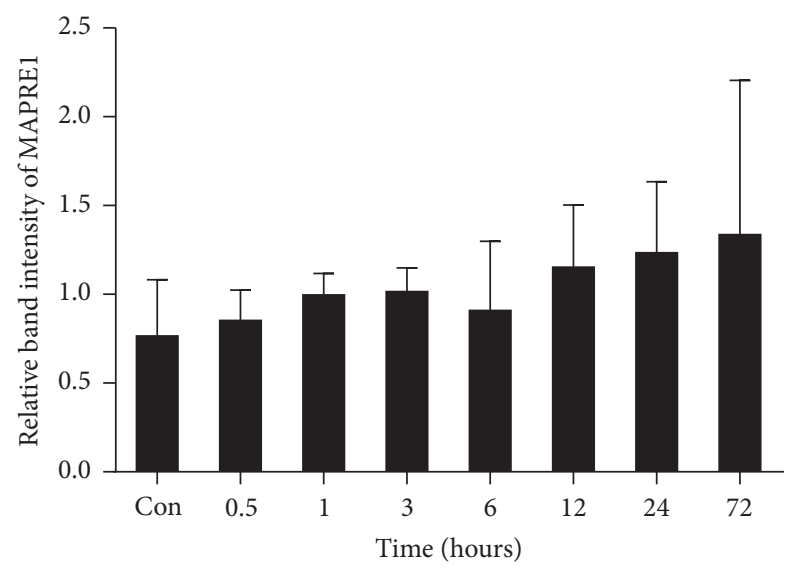

(b)

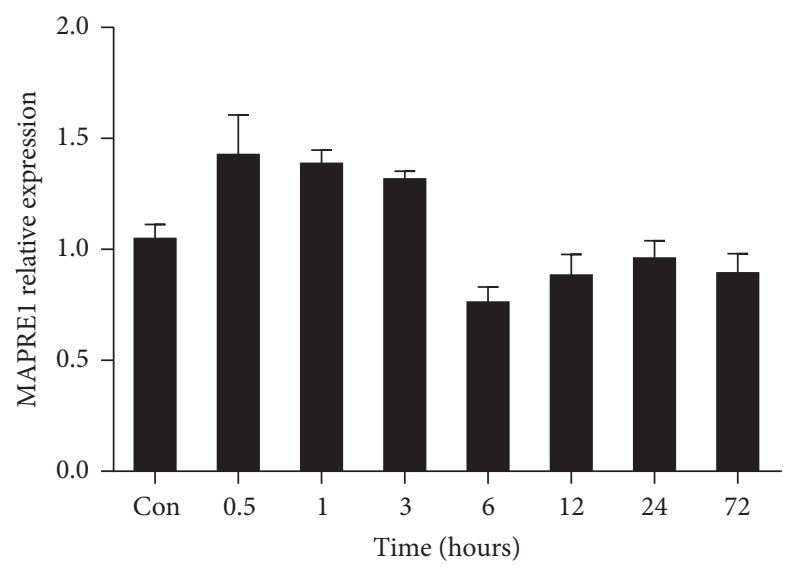

(c)

FIGURE 4: Representative western blot and RT-PCR assays of MAPRE1. (a) The first stripe was MAPRE1 protein; the other was $\beta$ actin as the internal stripe. (b) MAPRE1 protein relative expression. While there was no significant difference among the groups at each time point $(P=0.69)$, levels were lowest at IR/OGDR $6 \mathrm{~h}$. (c) MAPRE1 gene expression. While there was no significant difference among the groups $(P=0.634)$, gene expression was least at IR/OGDR $6 \mathrm{~h}$.

Evidence has shown that the rate of $\mathrm{Cx} 43$ degradation can be modulated at both the level of GJ endocytosis and postendocytic sorting of $\mathrm{Cx}$ to lysosomes [50]. The researchers provided molecular and mechanistic insights into GJ degradation, utilizing clathrin-mediated endocytosis components $[9,51]$. Cx43 may function not only in early noticeable invagination, but also in the process of degradation of this structure. It is suggested that dynamin interferes with successive stages of the internalization process [52].
In this study, we analyzed changes in Cx43 and its related mechanism by using laser-scanning confocal microscopy, western blot, and RT-PCR. This is conducive to evaluate comprehensively the effect of $\mathrm{Cx} 43$ on the structure and function of the brain, to reveal the role of $\mathrm{Cx} 43$ in ischemic stroke, and to provide new ideas for treatment. On the basis of our observations of the changes of $\mathrm{Cx} 43$ after ischemia, we analyzed its mechanism from the aspects of microtubule transport and endocytosis for an in-depth understanding of the factors that influence $\mathrm{Cx} 43$ functional changes and provide a theoretical basis for selecting a method of clinical intervention. These results indicate that $\mathrm{Cx} 43$ internalization may be considered a novel therapeutic target.

The internalization of GJs is a rarely reported mechanism. Although we demonstrated that Cx43 internalization was partially associated with endocytosis, the mechanisms of $\mathrm{Cx} 43$ internalization remain to be elucidated. Increasing evidence indicates that the process may involve phosphorylation and ubiquitination or polypeptide domains that act as sorting signals during ischemia/hypoxia [7, 53, 54]. Further studies are necessary to identify all the pathways responsible for $\mathrm{Cx} 43$ internalization.

Under conditions of ischemia, the amount of $\mathrm{Cx} 43$ did not change, but internalization of $\mathrm{Cx} 43$ occurred. This internalization was partly mediated through endocytosis, in response to the ischemic insult. Furthermore, internalization was not related to microtubule transport. The involvement of Cx43 within astrocytes during ischemic insults warrants study of the protective roles of astrocytes and gap conjunctions and provides a therapeutic target for treatment of brain ischemia.

\section{Conflicts of Interest}

The authors declare that they have no conflicts of interest.

\section{Acknowledgments}

This work was supported by Surface Project (Grant No. 81271293), the National Science Foundation for Young Scientists of China (Grant No. 81000490), and the Natural Science Foundation of Jilin Province (Grant Nos. 2010133 and 2015Y032-3).

\section{References}

[1] D. J. Rossi, J. D. Brady, and C. Mohr, "Astrocyte metabolism and signaling during brain ischemia," Nature Neuroscience, vol. 10, no. 11, pp. 1377-1386, 2007.

[2] S. Olk, G. Zoidl, and R. Dermietzel, "Connexins, cell motility, and the cytoskeleton," Cell Motility and the Cytoskeleton, vol. 66, no. 11, pp. 1000-1016, 2009.

[3] R. Dermietzel, E. L. Hertzberg, J. A. Kassier, and D. C. Spray, "Gap junctions between cultured astrocytes: immunocytochemical, molecular, and electrophysiological analysis," Journal of Neuroscience, vol. 11, no. 5, pp. 1421-1432, 1991.

[4] C. Giaume, C. Fromaget, A. El Aoumari, J. Cordier, J. Glowinski, and D. Grost, "Gap junctions in cultured astrocytes: singlechannel currents and characterization of channel-forming protein," Neuron, vol. 6, no. 1, pp. 133-143, 1991. 
[5] L. Matsuuchi and C. C. Naus, "Gap junction proteins on the move: connexins, the cytoskeleton and migration," Biochimica et Biophysica Acta-Biomembranes, vol. 1828, no. 1, pp. 94-108, 2013.

[6] D. W. Laird, "Life cycle of connexins in health and disease," Biochemical Journal, vol. 394, no. 3, pp. 527-543, 2006.

[7] J. L. Solan and P. D. Lampe, "Connexin43 phosphorylation: structural changes and biological effects," Biochemical Journal, vol. 419, no. 2, pp. 261-272, 2009.

[8] X. Wang, A. Ma, W. Zhu et al., "The role of connexin 43 and hemichannels correlated with the astrocytic death following ischemia/reperfusion insult," Cellular and Molecular Neurobiology, vol. 33, no. 3, pp. 401-410, 2013.

[9] M. M. Falk, S. M. Baker, A. M. Gumpert, D. Segretain, and R. W. Buckheit III, "Gap junction turnover is achieved by the internalization of small endocytic double-membrane vesicles," Molecular Biology of the Cell, vol. 20, no. 14, pp. 3342-3352, 2009.

[10] Y. Guo, C. Martinez-Williams, and D. E. Rannels, "Gap junction-microtubule associations in rat alveolar epithelial cells," American Journal of Physiology-Lung Cellular and Molecular Physiology, vol. 285, no. 6, pp. L1213-L1221, 2003.

[11] D. W. Laird, "The gap junction proteome and its relationship to disease," Trends in Cell Biology, vol. 20, no. 2, pp. 92-101, 2010.

[12] M. Z. Hossain, J. Peeling, G. R. Sutherland, E. L. Hertzberg, and J. I. Nagy, "Ischemia-induced cellular redistribution of the astrocytic gap junctional protein connexin 43 in rat brain," Brain Research, vol. 652, no. 2, pp. 311-322, 1994.

[13] E. E. Tansey, K. F. Kwaku, P. E. Hammer et al., "Reduction and redistribution of gap and adherens junction proteins after ischemia and reperfusion," Annals of Thoracic Surgery, vol. 82, no. 4, pp. 1472-1479, 2006.

[14] T. Shinotsuka, M. Yasui, and M. Nuriya, "Astrocytic gap junctional networks suppress cellular damage in an in vitro model of ischemia," Biochemical and Biophysical Research Communications, vol. 444, no. 2, pp. 171-176, 2014.

[15] S. F. Okada, L. Zhang, S. M. Kreda et al., "Coupled nucleotide and mucin hypersecretion from goblet-cell metaplastic human airway epithelium," American Journal of Respiratory Cell and Molecular Biology, vol. 45, no. 2, pp. 253-260, 2011.

[16] J. L. Perez Velazquez, L. Kokarovtseva, R. Sarbaziha, Z. Jeyapalan, and Y. Leshchenko, "Role of gap junctional coupling in astrocytic networks in the determination of global ischaemiainduced oxidative stress and hippocampal damage," European Journal of Neuroscience, vol. 23, no. 1, pp. 1-10, 2006.

[17] K. Jiang and A. Akhmanova, "Microtubule tip-interacting proteins: a view from both ends," Current Opinion in Cell Biology, vol. 23, no. 1, pp. 94-101, 2011.

[18] A. Akhmanova and M. O. Steinmetz, "Tracking the ends: a dynamic protein network controls the fate of microtubule tips," Nature Reviews Molecular Cell Biology, vol. 9, no. 4, pp. 309-322, 2008.

[19] S. D. Conner and S. L. Schmid, "Regulated portals of entry into the cell," Nature, vol. 422, no. 6927, pp. 37-44, 2003.

[20] G. J. Doherty and H. T. McMahon, "Mechanisms of endocytosis," Annual Review of Biochemistry, vol. 78, pp. 857-902, 2009.

[21] T. Kirchhausen, E. Macia, and H. E. Pelish, "Use of dynasore, the small molecule inhibitor of dynamin, in the regulation of endocytosis," Methods in Enzymology, vol. 438, pp. 77-93, 2008.

[22] M. W. Pfaffl, "A new mathematical model for relative quantification in real-time RT-PCR," Nucleic Acids Research, vol. 29, no. 9, p. e45, 2001.
[23] M. C. Fiori, L. Reuss, L. G. Cuello, and G. A. Altenberg, "Functional analysis and regulation of purified connexin hemichannels," Frontiers in Physiology, vol. 5, article 71, 2014.

[24] T. Nakase, Y. Yoshida, and K. Nagata, "Enhanced connexin 43 immunoreactivity in penumbral areas in the human brain following ischemia," GLIA, vol. 54, no. 5, pp. 369-375, 2006.

[25] M. Xie, C. Yi, X. Luo et al., "Glial gap junctional communication involvement in hippocampal damage after middle cerebral artery occlusion," Annals of Neurology, vol. 70, no. 1, pp. 121-132, 2011.

[26] T. Nakase, G. Söhl, M. Theis, K. Willecke, and C. C. G. Naus, "Increased apoptosis and inflammation after focal brain ischemia in mice lacking connexin 43 in astrocytes," American Journal of Pathology, vol. 164, no. 6, pp. 2067-2075, 2004.

[27] J. A. Orellana, D. E. Hernández, P. Ezan et al., "Hypoxia in high glucose followed by reoxygenation in normal glucose reduces the viability of cortical astrocytes through increased permeability of connexin 43 hemichannels," GLIA, vol. 58, no. 3, pp. 329-343, 2010.

[28] J. A. Orellana, N. Froger, P. Ezan et al., "ATP and glutamate released via astroglial connexin 43 hemichannels mediate neuronal death through activation of pannexin 1 hemichannels," Journal of Neurochemistry, vol. 118, no. 5, pp. 826-840, 2011.

[29] A. D. Martinez and J. C. Sáez, "Regulation of astrocyte gap junctions by hypoxia-reoxygenation," Brain Research Reviews, vol. 32, no. 1, pp. 250-258, 2000.

[30] X. Dang, B. W. Doble, and E. Kardami, "The carboxy-tail of connexin-43 localizes to the nucleus and inhibits cell growth," Molecular and Cellular Biochemistry, vol. 242, no. 1-2, pp. 35-38, 2003.

[31] D. V. Krysko, L. Leybaert, P. Vandenabeele, and K. D’Herde, "Gap junctions and the propagation of cell survival and cell death signals," Apoptosis, vol. 10, no. 3, pp. 459-469, 2005.

[32] J. A. Orellana, N. Palacios-Prado, and J. C. Sáez, "Chlorpromazine reduces the intercellular communication via gap junctions in mammalian cells," Toxicology and Applied Pharmacology, vol. 213, no. 3, pp. 187-197, 2006.

[33] D. C. Spray, R. Hanstein, S. V. Lopez-Quintero, R. F. Stout, S. O. Suadicani, and M. M. Thi, "Gap junctions and Bystander effects: good samaritans and executioners," Wiley Interdisciplinary Reviews: Membrane Transport and Signaling, vol. 2, no. 1, pp. 1-15, 2013.

[34] N. Rouach, M. Segal, A. Koulakoff, C. Giaume, and E. Avignone, "Carbenoxolone blockade of neuronal network activity in culture is not mediated by an action of gap junctions," Journal of Physiology, vol. 553, no. 3, pp. 729-745, 2003.

[35] M. V. Frantseva, L. Kokarovtseva, and J. L. Perez Velazquez, "Ischemia-induced brain damage depends on specific gapjunctional coupling," Journal of Cerebral Blood Flow and Metabolism, vol. 22, no. 4, pp. 453-462, 2002.

[36] Y. Leshchenko, S. Likhodii, W. Yue, W. M. Burnham, and J. L. Perez Velazquez, "Carbenoxolone does not cross the blood brain barrier: an HPLC study," BMC Neuroscience, vol. 7, article 3, 2006.

[37] V. Benfenati, M. Caprini, G. P. Nicchia et al., "Carbenoxolone inhibits volume-regulated anion conductance in cultured rat cortical astroglia," Revista de la Asociación Espaola de Neuropsiquiatría, vol. 3, pp. 323-336, 2009.

[38] P. Bazzigaluppi, I. Weisspapir, B. Stefanovic, L. Leybaert, and P. L. Carlen, "Astrocytic gap junction blockade markedly increases extracellular potassium without causing seizures in the mouse neocortex," Neurobiology of Disease, vol. 101, pp. 1-7, 2017. 
[39] J. C. Sáez, V. M. Berthoud, M. C. Brañes, A. D. Martínez, and E. C. Beyer, "Plasma membrane channels formed by connexins: their regulation and functions," Physiological Reviews, vol. 83, no. 4, pp. 1359-1400, 2003.

[40] R. M. Shaw, A. J. Fay, M. A. Puthenveedu, M. von Zastrow, Y.N. Jan, and L. Y. Jan, "Microtubule plus-end-tracking proteins target gap junctions directly from the cell interior to adherens junctions," Cell, vol. 128, no. 3, pp. 547-560, 2007.

[41] B. N. G. Giepmans, I. Verlaan, T. Hengeveld et al., "Gap junction protein connexin- 43 interacts directly with microtubules," Current Biology, vol. 11, no. 17, pp. 1364-1368, 2001.

[42] S. Olk, A. Turchinovich, M. Grzendowski et al., "Proteomic analysis of astroglial connexin 43 silencing uncovers a cytoskeletal platform involved in process formation and migration," GLIA, vol. 58, no. 4, pp. 494-505, 2010.

[43] N. J. Severs, K. S. Shovel, A. M. Slade, T. Powell, V. W. Twist, and C. R. Green, "Fate of gap junctions in isolated adult mammalian cardiomyocytes," Circulation Research, vol. 65, no. 1, pp. 22-42, 1989.

[44] K. Jordan, R. Chodock, A. R. Hand, and D. W. Laird, "The origin of annular junctions: a mechanism of gap junction internalization," Journal of Cell Science, vol. 114, no. 4, pp. 763773, 2001.

[45] G. Gaietta, T. J. Deerinck, S. R. Adams et al., "Multicolor and electron microscopic imaging of connexin trafficking," Science, vol. 296, no. 5567, pp. 503-507, 2002.

[46] G. G. Hesketh, M. H. Shah, V. L. Halperin et al., "Ultrastructure and regulation of lateralized connexin 43 in the failing heart," Circulation Research, vol. 106, no. 6, pp. 1153-1163, 2010.

[47] G. Scita and P. P. Di Fiore, “The endocytic matrix," Nature, vol. 463, no. 7280, pp. 464-473, 2010.

[48] A. M. Gumpert, J. S. Varco, S. M. Baker, M. Piehl, and M. M. Falk, "Double-membrane gap junction internalization requires the clathrin-mediated endocytic machinery," FEBS Letters, vol. 582, no. 19, pp. 2887-2892, 2008.

[49] S. Mayor and R. E. Pagano, "Pathways of clathrin-independent endocytosis," Nature Reviews Molecular Cell Biology, vol. 8, no. 8, pp. 603-612, 2007.

[50] E. Leithe, S. Sirnes, T. Fykerud, A. Kjenseth, and E. Rivedal, "Endocytosis and post-endocytic sorting of connexins," Biochimica et Biophysica Acta-Biomembranes, vol. 1818, no. 8, pp. 1870-1879, 2012.

[51] J. T. Fong, R. M. Kells, A. M. Gumpert, J. Y. Marzillier, M. W. Davidson, and M. M. Falk, "Internalized gap junctions are degraded by autophagy," Autophagy, vol. 8, no. 5, pp. 794-811, 2012.

[52] J. Gilleron, D. Carette, C. Fiorini et al., "The large GTPase dynamin2: a new player in connexin 43 gap junction endocytosis, recycling and degradation," International Journal of Biochemistry and Cell Biology, vol. 43, no. 8, pp. 1208-1217, 2011.

[53] D. W. Laird, "Connexin phosphorylation as a regulatory event linked to gap junction internalization and degradation," Biochimica et Biophysica Acta-Biomembranes, vol. 1711, no. 2, pp. 172-182, 2005.

[54] V. M. Berthoud, P. J. Minogue, J. G. Laing, and E. C. Beyer, "Pathways for degradation of connexins and gap junctions," Cardiovascular Research, vol. 62, no. 2, pp. 256-267, 2004. 

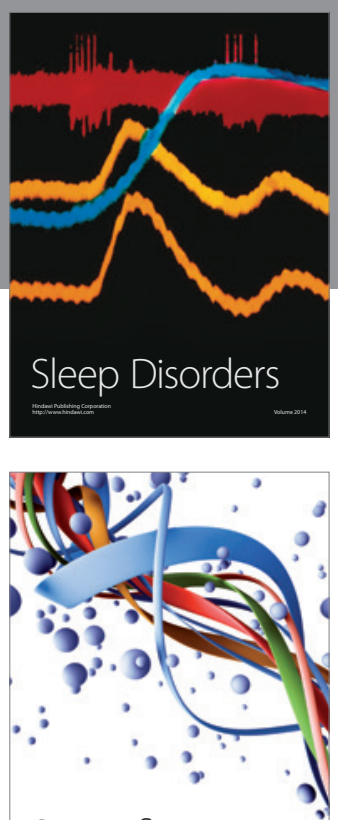

Scientifica
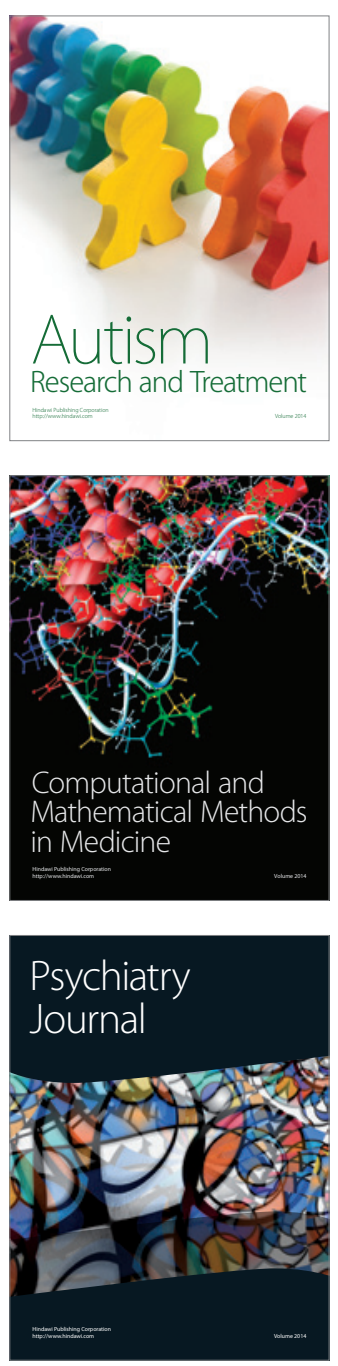
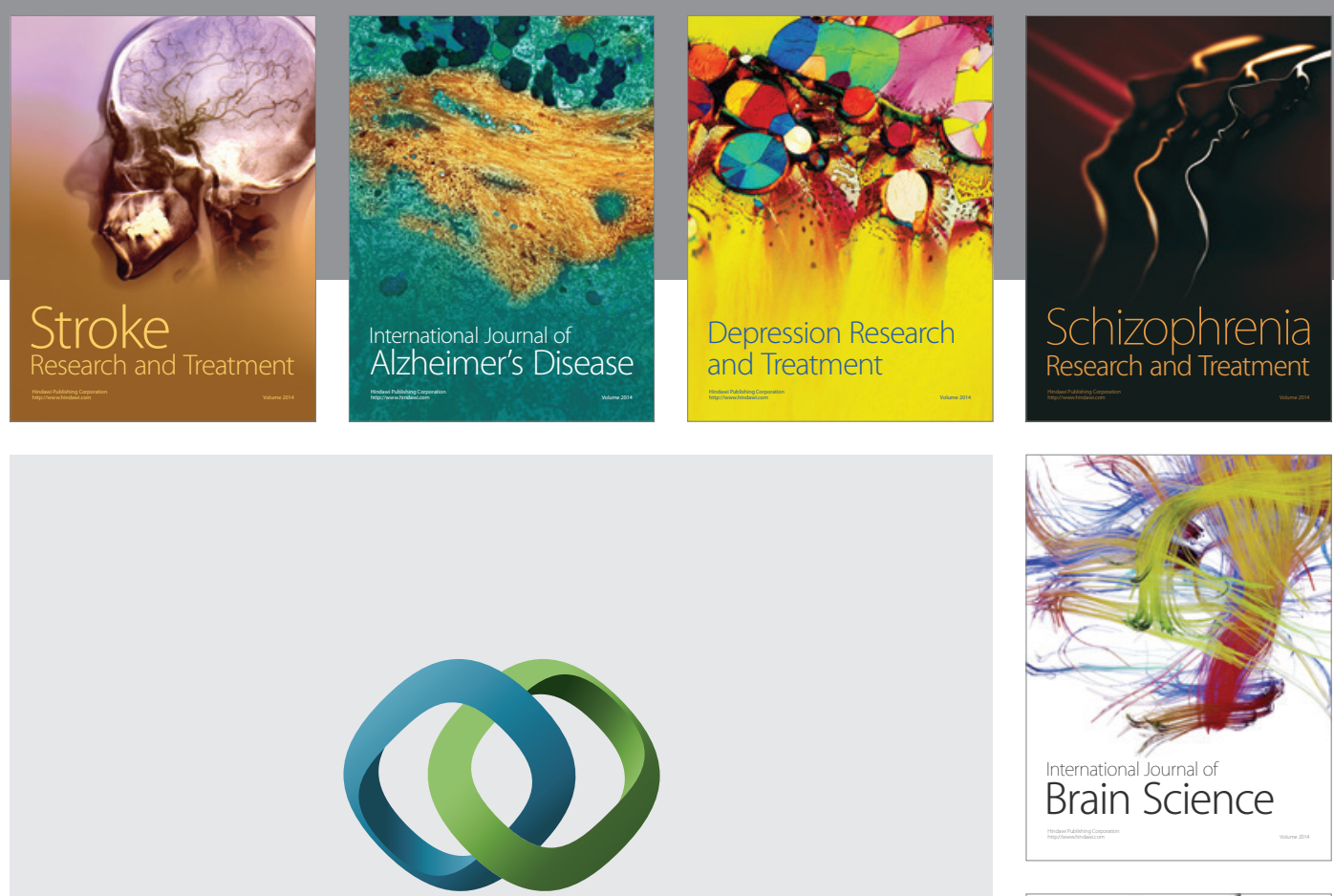

\section{Hindawi}

Submit your manuscripts at

https://www.hindawi.com
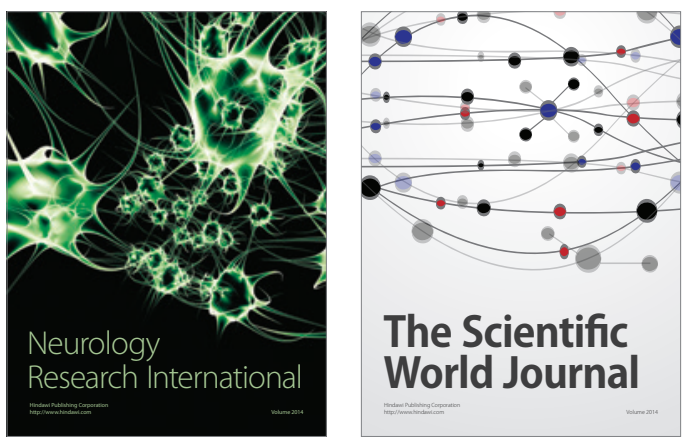

The Scientific World Journal

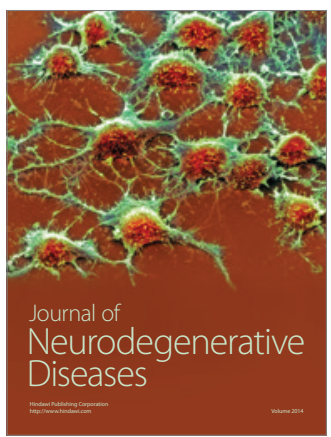

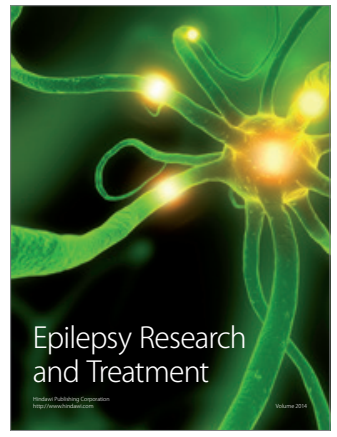

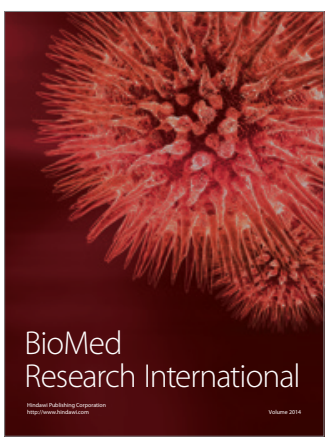

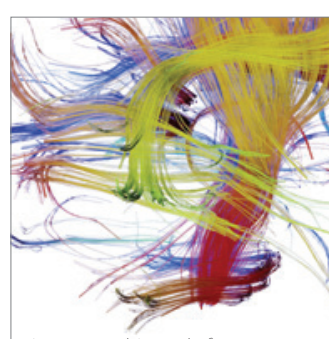

Brain Science

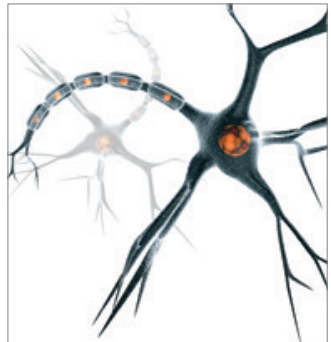

Neural Plasticity
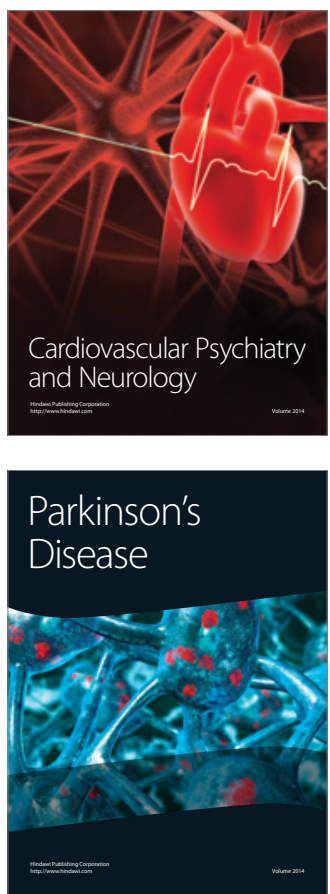